

EDITORIAL

\title{
EL BAJO PESO AL NACER: OTRO EJEMPLO DE INEQUIDAD EN COLOMBIA
}

\section{Low birth weight: another example of sociall inequity in Colombia}

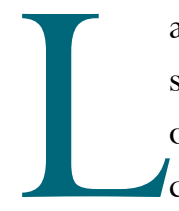

a mortalidad perinatal es un indicador de la salud materno-infantil que evalúa la calidad del control prenatal, y se considera una causa de muerte evitable ${ }^{1}$ que comprende las muertes fetales ocurridas entre la semana $22 \mathrm{y}$ el $7^{\circ}$ día de nacimiento. ${ }^{2}$ Dentro de la mortalidad perinatal, el bajo peso al nacer (BPN) tiene una gran importancia. Este problema afectó, según el Departamento Administrativo Nacional de Estadística (DANE), al 8,4\% de los Recién Nacidos (RN) vivos en Colombia en el año 2006. ${ }^{3}$ También afecta, de manera importante, la mortalidad en menores de 1 año ya que se halla estrechamente relacionada con los trastornos respiratorios específicos del período perinatal y las mal definidas "otras afecciones del período perinatal" que causan, respectivamente, $25 \%$ y $10 \%$ de la mortalidad en menores de un año en el país. ${ }^{4}$ En este orden de ideas, el bajo peso al nacer tiene un importante efecto sobre la carga de enfermedad para la sociedad colombiana. ${ }^{5}$

La solución de este problema, sin embargo, no parece estar a la mano a corto plazo debido a factores tales como: el subregistro, el análisis deficiente de los casos de mortalidad perinatal y las limitaciones que el sistema de salud actual tiene para brindar salud de manera equitativa a los colombianos.

Tanto la mortalidad perinatal como la mortalidad materna hacen parte de los protocolos de vigilancia epidemiológica en el país y se deben informar, de manera obligatoria, a los entes locales reguladores de salud. ${ }^{6}$ A pesar de la existencia de esta normatividad, es posible que se presente un subregistro importante. Por ejemplo, según el DANE, tanto el departamento de Antioquia como el de Chocó tienen una tasa de RN con peso menor a $2.500 \mathrm{~g}$ de 9,2\%; sin embargo, la tasa de mortalidad neonatal temprana es de 7,1 $\mathrm{x}$ $1.000 \mathrm{RN}$ vivos en Antioquia y de 17,4 x $1.000 \mathrm{RN}$ vivos para el Chocó. Al mismo tiempo, la proporción de la población en condiciones de miseria corresponde a 31\% en el Chocó y a 8,4\% en Antioquia. Por último, no hay información válida sobre el número de partos atendidos por personal calificado en los municipios del Chocó. ${ }^{7}$ Por lo tanto, es posible considerar la existencia del subregistro en varias de las regiones del país, especialmente en las más marginadas. Dicho subregistro ha sido reportado previamente en otros países. ${ }^{1}$

Los Comités de Vigilancia Epidemiológica (COVES) no tienen el alcance de los comités de revisión de mortalidad materna y perinatal en países como Nueva Zelanda ${ }^{8}$ o Australia. ${ }^{9}$ Estos comités son un instrumento de apoyo para quienes deben tomar las decisiones en la aplicación de intervenciones y políticas dirigidas a reducir el impacto de estas entidades en sus comunidades. ${ }^{7}$ Además, los COVES no tienen la capacidad suficiente para analizar todos los casos de mortalidad en fetos mayores de 1.000 g y de recién nacidos en los primeros 7 días de vida que se presentan a nivel regional. ${ }^{10}$ La metodología que se utiliza en el país en la actualidad adolece de serios defectos que limitan el conocimiento de las causas de la muerte perinatal. Por ejemplo, la autopsia perinatal, procedimiento indispensable para conocer los verdaderos orígenes de la mortalidad perinatal, sólo se hace en un número muy limitado de casos. ${ }^{8}$ Por último, entre algunos de los trabajadores de la salud existe la percepción de que estos comités funcionan 
más como tribunales en busca de culpables que como entes de mejoramiento de la calidad con una metodología sistemática.

Por otra parte, las complicaciones graves del feto y las que determinan el parto antes del término no son tan dramáticas como la hemorragia, la preeclampsia o la infección grave que afectan a la madre. El sistema no cuenta con alarmas que señalen atención pronta y oportuna ya sea a nivel comunitario, administrativo o institucional.

El ámbito de las Instituciones Prestadoras de Salud (IPS) es un lugar poco escrutado, donde no siempre se dispone de la estructura para la atención adecuada del parto o del recién nacido con complicaciones. En ocasiones, los procesos de atención son defectuosos. En algunas de estas instituciones existen regímenes de contratación lesivos para los trabajadores de la salud que, a su vez, afectan negativamente la calidad de la atención maternoinfantil debido a situaciones tales como la limitación en la disponibilidad de tiempo o la existencia de guías de atención que toman en cuenta únicamente criterios de contención de costos y no de efectividad clínica.

Tampoco se conoce el efecto que tienen sobre la mortalidad perinatal las barreras administrativas asociadas al sistema de aseguramiento que nos rige. Aún no se ha evaluado en la salud perinatal el impacto de la entrega inoportuna de autorizaciones, por parte de las aseguradoras, para la realización de: procedimientos diagnósticos en embarazos con morbilidad fetal asociada, intervenciones fetales o para la atención del recién nacido. Estas limitaciones se presentan de manera diferencial y afectan más al grupo de los mal llamados "vinculados" y a quienes pertenecen al régimen subsidiado. ${ }^{11}$

El trabajo realizado por un grupo de investigadores en el Hospital San José de Popayán muestra cómo los estratos sociales más bajos son los más afectados por el bajo peso al nacer. Como en el caso de la mortalidad materna, el bajo peso al nacer es un indicador de inequidad que afecta a los grupos de población más pobres.

Rudolf Virchow, reconocido por ser el fundador de la teoría de la patología celular, en 1848 dijo que "la medicina es una ciencia social, y la política no es sino medicina a gran escala”. Estas declaraciones que, aún a comienzos del siglo XXI, tienen inocultables resonancias, promueven la reflexión sobre los problemas de salud que, al igual que el bajo peso al nacer, permanecen sin la debida atención. ${ }^{12}$ Geoffrey Rose, el reconocido epidemiólogo británico, también afirmaba que vastas poblaciones pueden estar enfermas, como las madres de los estratos menos favorecidos y que, al contrario de la atención clínica del paciente individual, ofrecer una solución a esas poblaciones debe provenir de acciones políticas. ${ }^{12}$

Entre el personal médico y quienes deciden las políticas en salud predomina la idea de que la enfermedad es un asunto que concierne sólo a la persona enferma. Puede ser alejada la idea de que el sistema de salud y aún la sociedad padecen de enfermedad y por eso la solución de los problemas materno-infantiles, ligados estrechamente con las condiciones de vida y con la pobreza, se basa en un enfoque individual. En el caso del BPN, la eliminación de las desigualdades sociales es la manera más efectiva de prevenirlo. Los autores de la referida contribución original a nuestra revista, reclaman en la discusión justamente una política pública clara, con suficiente presupuesto y gestión frente al problema del BPN.

Si una parte de la sociedad padece de una enfermedad, toda la sociedad padece; el bienestar tiene como condición ser compartido, o no lo es. El bajo peso al nacer es, entonces, un problema social.

\section{Hernando Gaitán D., M.D., MSc}

Editor

\section{Mario Delgado N., M.D., MSc}

Editor invitado

\section{REFERENCIAS}

1. Freitas A, Puigdefâbregas A, Gispert R, Barés M, Bustins M. La mortalidad perinatal según 2 fuentes de información. Gac Sanit 2008;22:378-81.

2. Ministerio de la Protección Social. Instituto Nacional de Salud. Protocolo de vigilancia de la mortalidad perinatal. Colombia; 2003.

3. Departamento Administrativo Nacional de Estadística (DANE). Estadísticas Vitales. Nacimientos por peso al nacer. Visitado en 2009 Jun 17. Disponible en: http://www.dane.gov. $\mathrm{co} /$ index.php?option $=$ com_content\&task $=$ category\&sectio nid $=16 \&$ id $=393 \&$ Itemid $=889$ 
4. Ministerio de la Protección Social, Colombia. Situación de salud en Colombia: indicadores básicos 2007. Visitado en 2009 Jun 17. Disponible en: http://www. minproteccionsocial.gov.co/VBeContent/NewsDetail. asp $\mathrm{ID}=15895 \& \mathrm{IDCompany}=3$

5. MINSALUD. La carga de la enfermedad en Colombia. Bogotá; 1994.

6. Instituto Nacional de Salud. Subdirección de Vigilancia y Control. Protocolo de vigilancia de mortalidad perinatal. Bogotá (Colombia); 2007.

7. Porcentaje de atención del parto por personal calificado. Sitio oficial de Nuquí en Chocó (Colombia). Visitado en 2009 Jun 19. Disponible en: http://nuqui-choco.gov.co/ odm.shtml?apc $=a-e 1--\& x=2823038$

8. Perinatal and Maternal Mortality Review Committee 2007. First report to the minister of health June 2005-June 2007. Wellington Perinatal and Maternal mortality Review
Committee. Visitado en 2009 Jun 18. Disponible en: http:// www.pmmrc.health.govt.nz/moh.nsf/indexcm/pmmrcresources-first-report-200507?Open\&m_id=6.1

9. NSW Ministerial Maternal and Perinatal Committee. NSW Health. Visitado en 2009 Jun 18. Disponible en: http:/www. health.nsw.gov.au/health_pr/mph/mpc/

10. Sala de situación: mortalidad materna y perinatal en Santander. Visitado en 2009 Jun 16. Disponible en: http:// www.saludsantander.gov.co/observatorio/foros/Document o\%20Mortalidad\%20Materna\%20Perinatal.pdf

11. Instituto Nacional de Salud. Subdirección de Vigilancia y Control en Salud Pública. Primer COVE Nacional Materno Perineal. Bogotá; 2009.

12. Mackenbach JP. Politics is nothing but medicine at a larger scale: reflections on public health's biggest idea. J Epidemiol Community Health 2009;63:181-4. 